\title{
Transmisión de video simultaneo en ancho de banda limitado aplicando esteganografía
}

\author{
Julio C. Suarez-Tapia ${ }^{1}$, Blanca E. Carvajal-Gámez ${ }^{1,2}$, \\ Chadwick Carreto-Arellano ${ }^{1}$ \\ ${ }^{1}$ Instituto Politécnico Nacional, \\ Unidad Profesional Interdisciplinaria de Ingeniería y Tecnologías Avanzadas, \\ México DF \\ ${ }^{2}$ Instituto Politécnico Nacional, Escuela Superior de Cómputo, \\ SEPI-ESCOM, México DF \\ \{jcsuarez, becarvajal\}@ipn.mx
}

\begin{abstract}
Resumen. El problema de la transferencia de datos en medios limitados en ancho de banda, y la seguridad en la transferencia de estos, que en algunos casos como los medios inalámbricos deben de considerarse y administrarse sus recursos, han generado diversas propuestas de solución para este problema, sobre todo en infraestructura y en sistemas de administración de recursos. Sin embargo, en algunos casos como en infraestructura se invierte mucho tiempo y dinero. En este trabajo se presenta un algoritmo esteganográfico en video sin audio en formato MPEG-4, para la transferencia de información de otro video de igual formato. El objetivo de este trabajo es presentar una doble propuesta de solución a estas limitaciones. Logrando el envío de video estaremos ahorrando ancho de banda del medio, así como logrando la aplicación de una técnica de seguridad sobre la información enviada. La propuesta consiste en aplicar un kernel de $3 \times 3$ para la estimación del campo de varianza en cada imagen, aunado a la descomposición wavelet Haar para poder detectar las zonas ruidosas de la secuencia de video e insertar los datos correspondientes de las imágenes del video a ocultar. Los resultados presentados están en términos cuantitativos y cualitativos de la imagen. Verificamos la calidad del algoritmo con las métricas del PSNR, NCD,MAE, Q, RMS, así como los histogramas que muestran que no hay alteración de la distribución de color en las imágenes.
\end{abstract}

Palabras clave: esteganografía, ancho de banda, recursos limitados, video, wavelet, Haar, MPEG-4.

\section{Introducción}

Actualmente aumenta la demanda de utilización de nuevos servicios inalámbricos, como los de sistemas de comunicaciones móviles, redes de difusión de televisión digital terrestre o los diversos sistemas de acceso inalámbrico de banda ancha, como el enlace local inalámbrico, conocida como la conexión de última milla [1,2]. Con la rápida 
evolución tecnológica y el fenómeno de la convergencia, de los contenidos multimedia y de los dispositivos electrónicos, se está creando un entorno dinámico, es indispensable para el desarrollo de una adecuada y oportuna planificación y eficaz gestión, a los fines de optimizar su uso del ancho de banda. Los dispositivos móviles tienen muchas restricciones y limitaciones en relación al consumo de la energía en comparación con los dispositivos de una red cableada [1,2]. La conservación de la energía de ambientes inalámbricos es un asunto importante que debe tomarse en cuenta. Las redes de este tipo están expuestas a muchos factores que contrarrestan el uso óptimo de la energía, tales como la continua comunicación entre los dispositivos, alojamiento de recursos y memoria, el uso eficiente de la batería de alimentación, el tráfico, etc. [3]

El uso eficiente de recursos energéticos tiene como objetivos la viabilidad económica, es decir que la tecnología sea económica; responsabilidad social mediante la construcción de tecnología que contribuya a minimizar los problemas de consumo irracional de energía y minimizar el impacto en el ambiente. En el contexto del cómputo móvil, redes inalámbricas y telefonía inalámbrica, se han realizado múltiples esfuerzos por optimizar el uso de recursos, tales como el caudal de comunicación y la energía.

Ejemplo de esto ha sido la telefonía celular, las redes ManNets y las redes Ad Hoc, cuya utilidad en sus inicios ha sido restringida por la capacidad limitada de almacenamiento de energía y mínimas optimizaciones para su administración. Por tal motivo, en el contexto de las redes inalámbricas, las optimizaciones han sido enfocadas a minimizar el número de dispositivos activos y de mensajes transmitidos por difusión, tomando como criterios de calidad el mantenimiento de conectividad y calidad en el servicio (QoS) en los flujos de comunicación [1].

Minimizar el número de dispositivos activos es logrado a través de la elección de un conjunto dominante de comunicación, es decir, un subconjunto mínimo de dispositivos que actúen con suficiente capacidad de almacenamiento de mensajes y energía [1]. El coste para desplegar una red inalámbrica es normalmente dominado por gastos de bienes inmuebles, planificación, mantenimiento, red de distribución, energía, etc. Por consiguiente crear la infraestructura de una red inalámbrica con pocos recursos es complicado, en lugares como las escuelas que hay muchas solicitudes a servicios multimedia el coste de desplegado en dispositivos móviles aumenta $[1,2]$.

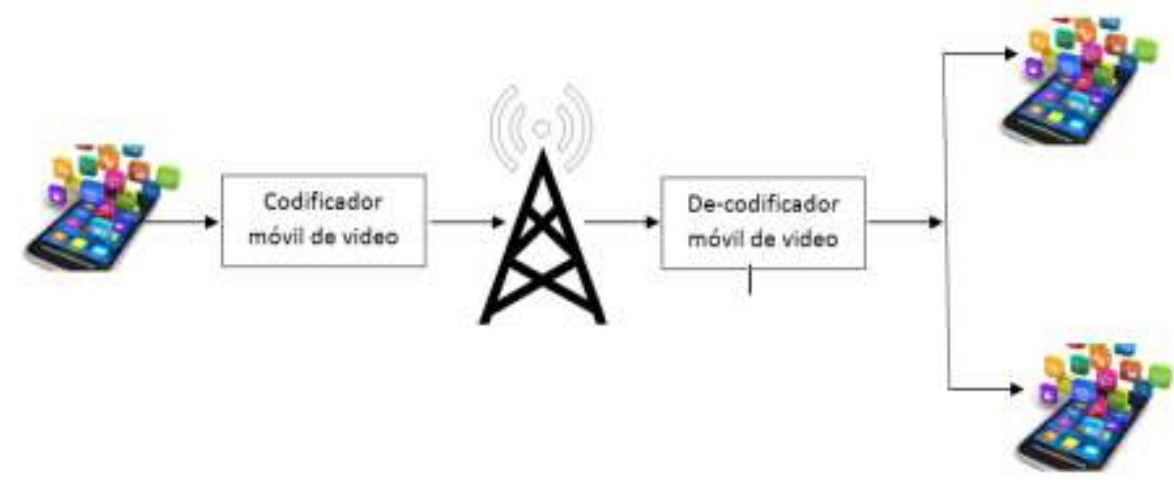

Fig. 1. Arquitectura del sistema propuesto.

Por consiguiente proponemos el uso de los algoritmos esteganográficos como una herramienta alterna a la ya utilizada comúnmente como protección intelectual, a una 
herramienta eficaz del uso de la banda ancha en lugares donde las solicitudes de información son interrumpidas por el número de usuarios conectados. Proponiendo la siguiente arquitectura de solución colaboramos en una solución alterna para la transmisión y descarga de video de manera simultánea en dispositivos móviles, Figura 1.

Este trabajo está dividido en las siguientes secciones: sección 2 materiales y métodos, sección 3 teoría y cálculos, sección 4 discusión y sección 5 conclusiones.

\section{Materiales y métodos}

El ocultamiento de la información consiste en el proceso de integrar información o datos dentro de archivos digitales como: música, video e imágenes; estos archivos son conocidos como archivo anfitrión (host) [4]. Esta técnica es útil para el envío de información altamente confidencial como información de negocios y/o información personal durante la comunicación multimedia. En un sistema de imagen oculta, la imagen usada para llevar la información es llamada imagen anfitriona (cover image o host image). La imagen resultante, la cual ya se le inserto la información secreta deseada es conocida como estego-imagen [4-6]. Una de los resultados esperados es que la estego-imagen sea perceptualmente idéntica a la imagen portadora, con el fin de que los datos insertados no sean expuestos de manera tan fácil por el medio de transmisión. Posteriormente la estego-imagen se envía al receptor de la información [4-6]. Entonces finalmente el receptor podrá extraer la información oculta mediante la generación de una llave. Los datos ocultos son imperceptibles e imposibles de extraer por receptores no deseados o que no cuenten con la llave. La esteganografía en nuestros días es una técnica que se ha ido convirtiendo poco a poco en una herramienta de vital importancia para la protección de los derechos de autor, la cual permite un proceso de autentificación, el cual permite la distribución y uso legal de diferentes tipos de materiales. Una técnica esteganográfica es usualmente evaluada en términos de la calidad visual, de la capacidad de inserción, la calidad y porcentaje de la recuperación de los datos insertados; en otras palabras, un algoritmo esteganográfico ideal debe de tener una gran capacidad de ocultamiento y una excelente calidad visual del estegoobjeto generado. La manera más razonable para hacer frente a esta disyuntiva es proponer técnicas que puedan proporcionar un equilibrio entre ambas $[3,7,8]$, sin apartar de la vista el hecho de que se debe de recuperar totalmente los datos insertados en la estego-imagen. A juzgar por el hecho de la sensibilidad de la visión humana la cual debe de ser considerada en el diseño del algoritmo esteganográfico, los podemos categorizar los esquemas en tres tipos: (1) Los sistemas de alta capacidad de la inserción con calidad de imagen aceptable [4], (2) los regímenes de calidad de imagen alta con una capacidad moderada de inserción [4], y esquemas de alta eficiencia de inserción [4] con una ligera distorsión [4]. Por lo general, hay algunas técnicas para realizar 
estego-imagen como: en el dominio del espacio, dominio de la frecuencia y métodos adaptativos (toman las mejores cualidades de los otros dominios y trabajan en ellos simultáneamente). El método más común para el dominio espacial es la modificación del bit menos significativo (LSB), el cual consiste en modificar el bit menos significativo en cada pixel de la imagen [4]. En el dominio de la frecuencia, se encuentra la transformada discreta de Fourier (DFT), la transformada del coseno discreto (DCT), y la transformada wavelet discreta (DWT), se utilizan para transformar los valores de los píxeles espaciales en coeficientes de frecuencia [4]. El ojo humano es menos sensible al ruido en la sub-banda de altas frecuencias $[5,6]$. En aplicaciones donde el dominio de la frecuencia está implicado, dependiendo de la naturaleza de la imagen utilizada como portador, esta imagen puede ser alterada significativamente cuando se aplica un filtro digital como detectores de ruido. En esta investigación proponemos un algoritmo esteganográfico adaptativo montado sobre un dispositivo móvil, el cual va a ser utilizado como codificador para realizar la transmisión de 2 videos simultáneos sin audio. El estego-video deberá de llegar al receptor, donde también es un dispositivo móvil que servirá como receptor-decodificador del estego-video. El algoritmo esteganográfico propuesto y la manipulación del video es descrito en esta sección. El esquema propuesto consta de las siguientes etapas, Figura 2.

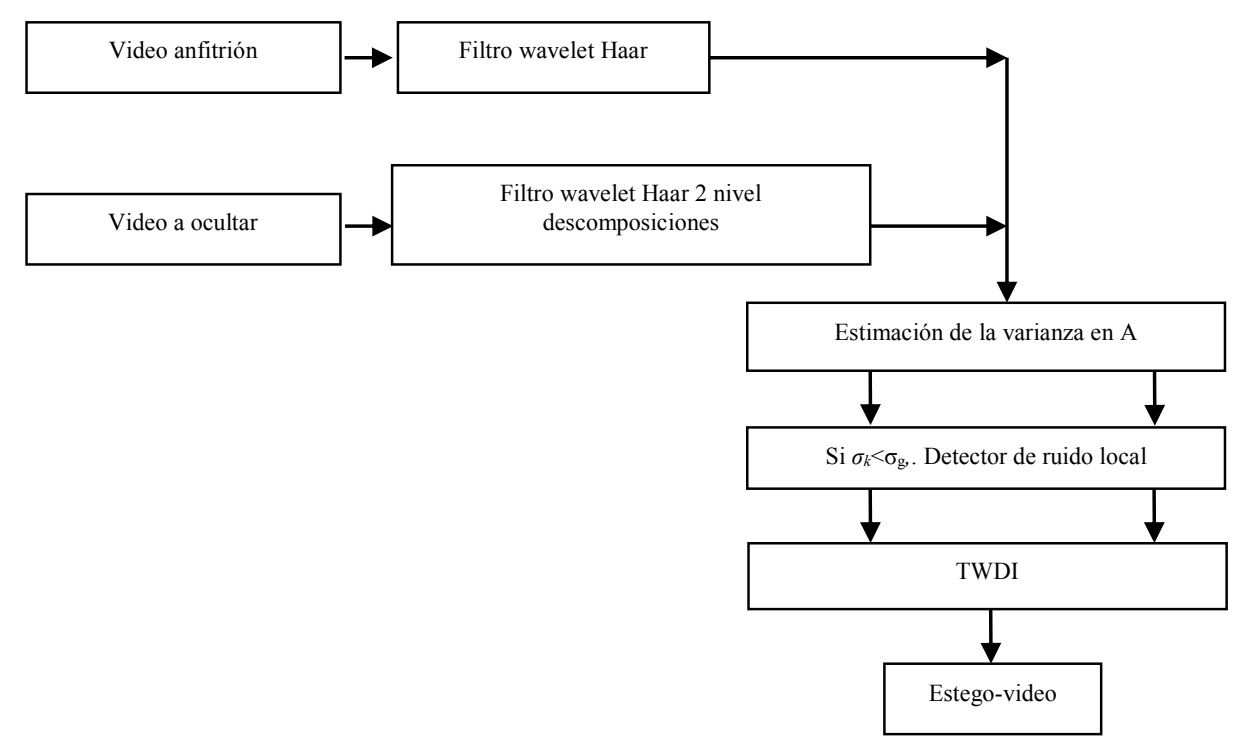

Fig. 2. Diagrama a bloques del algoritmo esteganográfico propuesto.

Este algoritmo consta de 5 etapas que serán explicadas a continuación:

Etapa de adquisición de video: El video adquirido consta de hasta 30 frames/segundo, es decir; 30 imágenes por segundo son transmitidos en un video de 5 
minutos en un formato *.mp4. MPEG-4 es una de las técnicas más recientes de compresión de video y permite relaciones de compresión mucho menor que el pasado MPEG-2. MPEG-4 es ideal para aplicaciones de bajo ancho de banda, exactamente como se requiere para la transmisión de video en una red inalámbrica, separando el video en imágenes se puede analizar cada imagen por separado, para poder hacer la inserción de la información. La descomposición se realiza tanto en el video anfitrión como en el video a ocultar $[7,8]$.

Etapa de descomposición wavelet: El algoritmo esteganográfico propuesto se representa en la Figura 1. Este algoritmo se aplica en cada canal de la imagen RGB del video portador. De la Figura 1, el bloque de la redundancia de los enfoques utiliza un nivel de descomposición con la wavelet Haar. Esta transformada, una de sus características es que suaviza la imagen portadora a través de una operación de convolución doble (primero de descomposición, y después de la reconstrucción) de los coeficientes de aproximación (A), detalles horizontales (DH), detalles diagonales (DD) y detalles verticales (DV). Así en las muestras de la imagen anfitriona $[5,6]$, proporciona mayor capacidad de ocultamiento y preservación de los detalles de la imagen [9-12].

Etapa de estimación simple del campo de varianza (ESCV): Para hacer el cálculo de la estimación simple del campo de varianza en la matriz de horizontales obtenida en la descomposición wavelet de la imagen portadora, se realiza el cálculo de la varianza general $\left(\sigma_{\mathrm{g}}\right)$ de cada sub-imagen [6],

$$
\sigma_{g}=\sqrt{\sum_{m=1}^{N}\left(y_{m}-y\right)^{2} / N},
$$

donde $y_{m}$ es el m-esimo elemento de la sub-imágen portadora, $y=\sum_{m=1}^{n} y_{m} / N$ es el valor medio de la actual imagen portadora, y $N$ es el número total de elementos en la sub-imagen portadora. Para el cálculo de la estimación local actual, se aplica una ventana de $3 \times 3$ sobre la doble descomposición wavelet Haar de la sub-imagen del video a ocultar [6],

$$
\sigma_{v}=\sqrt{\sum_{i=1}^{n}\left(x_{i}-x\right)^{2} / n},
$$

donde $x_{i}$ es el i-esimo elemento del actual kernel de la sub-imagen a ocultar, $x=\sum_{i=1}^{n} x_{i} / n$ es el valor medio del actual kernel, y $n=9$ es el número de elementos en la muestra del kernel. Obtenidos los valores de la estimaciónlocal y global del campo de varianza para cada uno de las imágenes del video portador como del video a ocultar entonces, se propone el siguiente umbral $\sigma_{k}<\sigma_{g}$.

Formalizando: "Si la estimación simple local del campo de varianza de la imagen a ocultar es menor a la estimación simple global del campo de varianza de la imagen 
portadora se oculta la información correspondiente". Con este criterio garantizamos la localización de las zonas ruidosas correspondientes.

Etapa de obtención de la estego-imagen: Para la obtención del estego-video, se van uniendo las estego-imágenes en un archivo alterno, donde se aplica la transformada wavelet discreta inversa en cada una de las imágenes portadoras para poder integrar finalmente el video MPEG-4, y así hacer el envío del estego-video.

Etapa de de-codificación del estego-video: Para poder recuperar los datos insertados en el estego-video, se sigue el mismo procedimiento realizado para la inserción, aplicando la condición siguiente: $\sigma_{k}<\sigma_{g}$. Finalmente se aplica dos niveles de recuperación de la transformada inversa utilizando la wavelet Haar.

\section{Resultados}

Los criterios usados para comparar esta relación de calidad con la imagen sin modificar son: la relación señal a ruido pico (PSNR), el error medio absoluto (MAE), la correlación (COI), el índice de calidad (Q), la desviación de color normalizada (NCD), y la capacidad de inserción (HC). Las pruebas se realizan en cada imagen que compone el video para finalmente obtener un promedio final de desempeño del algoritmo esteganográfico propuesto. La más común medida de distorsión es el PSNR [13],

$$
\text { PSNR }=10 \cdot \log \left[\frac{(255)^{2}}{\mathrm{MSE}}\right], \mathrm{dB},
$$

donde, $\quad \mathrm{MSE}=\frac{1}{M_{1} M_{2}} \sum_{i=1}^{M_{1} M_{2}} \sum_{j=1}|y(i, j)-x(i, j)|_{L_{2}}^{2}$ es el error medio cuadrático, donde $M_{1}, M_{2}$ son las dimensiones de la imagen, $y(i, j)$ es el vector 3D de los valores del pixel $(i, j)$ de la estego-imagen, $x(i, j)$ es el correspondiente pixel en la imagen anfitriona, y $\left\|_{L_{1}},\right\| \quad \|_{L_{2}}$ L1-y L2- son los vectores normales, respectivamente. La desviación del color normalizado (NCD) es usado para la cuantificación del error del color perceptual [13],

$$
\mathrm{NCD}=\frac{\sum_{i=1}^{M_{1} M_{2}}\left\|\Delta E_{L u v}(i, j)\right\|_{L_{2}}}{\sum_{i=1}^{M_{1} M_{2}} \sum_{j=1} \mid E_{L u v}^{*}(i, j) \|_{L_{2}}}
$$

Aqui, $\|\left.\Delta E_{L u v}(i, j)\right|_{L_{2}}=\left[\left(\Delta L^{*}(i, j)\right)^{2}+\left(\Delta u^{*}\right)^{2}+\left(\Delta v^{*}\right)^{2}\right]^{2 / 2}$ es la norma del error del color; $\Delta L^{*}, \Delta u^{*}, \mathrm{y} \Delta v^{*}$ son las diferencias en los componentes $L^{*}, u^{*}, \mathrm{y} v^{*}$, 
respectivamente, entre los dos vectores de color que representa a la estego-imagen y la imagen anfitriona para cada pixel $(i, j)$ de una imagen, y $\left\|E_{L u v}^{*}(i, j)\right\|_{L_{2}}=\left[\left(L^{*}\right)^{2}+\left(u^{*}\right)^{2}+\left(v^{*}\right)^{2}\right]^{1 / 2}$ es la norma o magnitud del pixel de la imagen anfitriona en el espacio $L^{*} u^{*} v^{*}$; El índice de calidad (Q) provee la calidad de la estegoimagen [13],

$$
\mathrm{Q}=\frac{4 \sigma_{x y} x y}{\left(\sigma_{x}^{2}+\sigma_{y}^{2}\right)\left(x^{2}+y^{2}\right)}
$$

donde $x$ y $y$ son los valores medios de la imagen anfitriona y de la estego-imagen, respectivamente, $\sigma_{x}^{2}$ y $\sigma_{y}^{2}$ son las varianzas de la imagen anfitriona y de la estegoimagen, respectivamente, y $\sigma_{x y}=\frac{1}{N-1} \sum_{i}^{N}\left(x_{i}-x\right)\left(y_{i}-y\right)$.La capacidad de inserción (HC), determina el número de bits que pueden insertarse en la imágen portadora [5, $6,13]$,

$$
H C=M S E \times \frac{\text { number of samples in embedding band }}{\text { number of bits of secure data }} .
$$

Las pruebas se realizaron sobre dos videos calidad MPEG-4, con una velocidad de 30 frames/segundo, y una tasa de 4Mbits/segundo, estándar establecido por la norma MPEG-4 para el video anfitrión, y para el video a ocultar presenta una velocidad de 30 imágenes/segundo con una tasa también de 4Mbits/segundo. Las tablas 1 y 2 muestran los resultados comparativos del estego-video, comparándolo con técnicas clásicas de esteganografía, como la LSB, la optimización del LSB (LSBO) [14,15]. A partir de estos resultados podremos validar si la técnica propuesta cumple con los tres compromisos básicos de las técnicas esteganográficos: i) capacidad de inserción, ii) calidad en la estego-imagen y iii) recuperación total de la imagen oculta. En la tabla 1 y 2 se muestra los resultados de desempeño del algoritmo propuesto en términos de PSNR, MAE, COI, Q, NCD y en HC para la tabla 1.

Tabla 1. Resultados comparativos de desempeño del algoritmo propuesto

\begin{tabular}{|c|c|c|c|c|c|}
\hline \multirow[b]{2}{*}{ Criterios } & \multicolumn{5}{|c|}{ Algoritmos esteganográficos } \\
\hline & 4LSB & 3LSB & 4LSBO & $3 \mathrm{LSBO}$ & $\begin{array}{c}\text { Estimación } \\
\text { simple del } \\
\text { campo de } \\
\text { varianza }\end{array}$ \\
\hline PSNR (dB) & 36.168 & 36.169 & 30.111 & 30.112 & 54.7468 \\
\hline MAE & 2.9911 & 2.9902 & 9.7307 & 9.7315 & 0.0721 \\
\hline CORR & 99.78 & 99.78 & 99.76 & 99.76 & 99.9990 \\
\hline Q & 0.9976 & 0.9976 & 0.9948 & 0.9948 & 0.9997 \\
\hline NCD & 0.00073 & 0.00073 & 0.0022 & 0.0022 & $6.1616 \mathrm{e}-6$ \\
\hline $\mathrm{HC}(\mathrm{KB})$ & 51,200 & 38,400 & 51,200 & 38,400 & 183,911 \\
\hline
\end{tabular}
para el estego-video con el video a ocultar. 
En la tabla 1 se puede observar que el algoritmo aquí propuesto cumple con los tres compromisos marcados para los algoritmos esteganográficos, si lo comparamos con los otros métodos mostrados podemos observar que la propuesta presenta: mayor capacidad de ocultamiento, mejor calidad de la estego-imagen que conforma el estegovideo, y para el último compromiso podemos observar que la calidad de la imagen recuperada en términos de Q y COI supera a las métodos tomados de comparativos, sin embargo para el MAE se encuentra con un valor intermedio entre el LSB y el LSBO, para el PSNR se encuentra en segundo lugar después del 3LSB.

Tabla 2. Resultados comparativos del video recuperado.

\begin{tabular}{|c|c|c|c|c|c|}
\hline \multirow[b]{2}{*}{ Criterios } & \multicolumn{5}{|c|}{ Algoritmos esteganográficos } \\
\hline & 4LSB & 3LSB & 4LSBO & $3 \mathrm{LSBO}$ & $\begin{array}{c}\text { Estimación } \\
\text { simple del } \\
\text { campo de } \\
\text { varianza }\end{array}$ \\
\hline PSNR (dB) & 28.2910 & 29.1210 & 28.5090 & 28.144 & 28.7083 \\
\hline MAE & 2.7469 & 2.7479 & 8.1255 & 8.1255 & 4.5247 \\
\hline CORR & 99.26 & 99.25 & 99.24 & 99.17 & 99.34 \\
\hline$Q$ & 0.9926 & 0.9922 & 0.9924 & 0.9920 & 0.9950 \\
\hline NCD & 0.0278 & 0.0203 & 0.0335 & 0.0332 & 0.0190 \\
\hline
\end{tabular}

Finalmente en la Figura 3 se muestra la imagen de error de la estego-imagen con la imagen anfitriona.

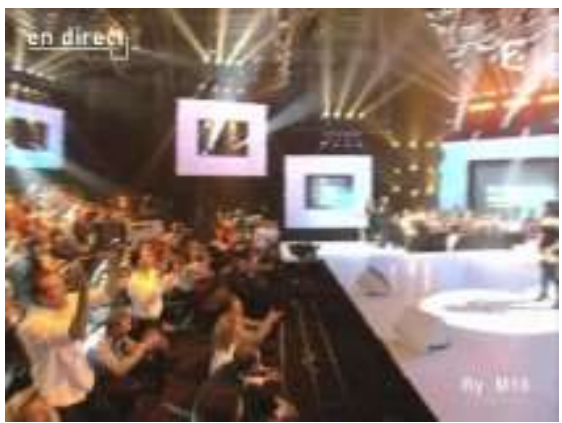

a)

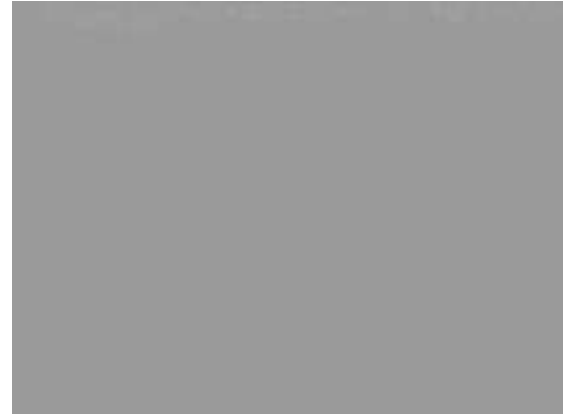

b)

Fig. 3. a) estego-imagen, b) imagen error obtenido a partir de la imagen original con la estego-imagen.

Para poder validar el envío simultáneo de video, para este caso de estudio se cálculo el ancho de banda $(\mathrm{AB})$ consumido por la transmisión del video anfitrión sobre un canal de transmisión, se toma como escenario real la situación de una cableada LAN, del Instituto Politécnico Nacional (IPN), el cual en horas picos de tráfico demora el envío y recepción de archivos multimedia, para el cálculo del AB se obtiene a partir de [16],

$A B=$ Velocidad $x$ tamaniodela imagen del video promedio $x$ porcentaje de actividad $x 8$ 
Donde velocidad esta dado por el número de frames/segundo, tamaño de la imagen es el estándar marcado por la norma MPEG-4 que es de 720x560 para este caso, porcentaje de actividad es en horas pico de hasta el $80 \%$. Así que obtenemos el siguiente resultado, para un video que dure 120 minutos con las características marcadas por la norma,

Tabla 3. Resultados comparativos del video recuperado.

\begin{tabular}{|c|c|c|c|c|c|}
\hline \multirow{2}{*}{$\begin{array}{c}\text { Características de } \\
\text { video }\end{array}$} & \multicolumn{5}{|c|}{ Aprovechamiento de Ancho de Banda } \\
\hline & Video 1 & Video 2 & Video 3 & & Total \\
\hline $\begin{array}{l}\text { Velocidad } \\
\text { (Kb/seg) }\end{array}$ & 240 & 360 & 240 & ---- & \\
\hline Frames/Segundo & 30 & 30 & 30 & ---- & \\
\hline $\begin{array}{l}\text { Porcentaje de } \\
\text { actividad(\%) }\end{array}$ & 80 & 78 & 90 & ---- & \\
\hline $\begin{array}{c}\text { AB total por } \\
\text { transmisión de } \\
\text { video }\end{array}$ & $1.57 \mathrm{Mbps}$ & $2.30 \mathrm{Mbps}$ & $1.769 \mathrm{Mbps}$ & $=$ & $5.56 \mathrm{Mbps}$ \\
\hline
\end{tabular}

Este resultado se multiplica por el número de usuarios conectados a la red por hora. Así que si tenemos una población de 500 usuarios máximos efectivos conectados, el $\mathrm{AB}$ necesario para satisfacer será la máxima demanda presentada en la tabla 3 por 500 usuarios, dando un total de 1,150 Mbps. Actualmente el IPN no cuenta con esta infraestructura para satisfacer esta demanda y su población va en aumento. Para la propuesta de solución en esta investigación si se toma el Video 2 como video portador, con esta técnica al menos podría enviarse simultáneamente otro video u otro tipo de datos multimedia ocupando solamente el AB calculado de $2.30 \mathrm{Mbps}$, sin llegar a ocupar los 1,150 Mbps obtenidos por 500 usuarios en hora pico.

\section{Discusión}

El algoritmo esteganográfico aquí propuesto para la transmisión simultánea de video en un ancho de banda limitado, dio resultados satisfactorios. Estos servirán para poder ocultar datos multimedia con una capacidad para la portadora de hasta $183,911 \mathrm{~KB}$. Este algoritmo se irá perfeccionando para mejorar la recuperación de la información oculta, para así mejorar el PSNR de esta. De los resultados experimentales expuestos en este artículo, se puede ver que el método propuesto provee los tres compromisos básicos de los algoritmos esteganográficos que son: capacidad de inserción, calidad de la estego-imagen y la recuperación de la información oculta. Los resultados cuantitativos podemos observar que se tiene de hasta un $99.9 \%$ de correlación de la imagen original vs estego-imagen, de igual manera se observa que para la calidad de la imagen se conserva hasta un $99.9 \%$ de la calidad visual que se puede ver en la Figura $3 \mathrm{~b}$ ), que se presenta la imagen de error de la estego-imagen y de la imagen a ocultar. Se tendrán que ir haciendo más pruebas para validar este método propuesto en diferentes escenarios, hasta este momento se ha validado la descarga simultanea del 
video anfitrión que contiene la información oculta, disminuyendo así en horas picos el consumo de ancho de banda limitado para este caso de estudio.

\section{Conclusión}

El método propuesto para el ocultamiento de archivos multimedia en anchos de banda limitado, usa la desviación estándar general y particular de un kernel de $3 \times 3$ como detectores de ruidos en imágenes, en conjunto aplicando la descomposición de los filtros wavelet Haar proveen una mayor eficiencia de los recursos en medios limitados para así tener un mayor aprovechamiento de estos como lo es para este caso de estudio del ancho de banda limitado y que exista alta demanda de solicitudes en horas pico. Se presenta un compromiso integral entre el procesamiento del video portador descomponiéndolo en imágenes que sirven de anfitrionas a información multimedia. Los resultados obtenidos avalan estos compromisos anteriormente mencionados que son: calidad de la imagen, capacidad de ocultamiento y recuperación de la información insertada en el video anfitrión. Al obtener de manera satisfactoria este compromiso se puede aplicar en redes limitadas para el máximo aprovechamiento del ancho de banda total.

Agradecimientos. El equipo de trabajo agradece al IPN y a CONACYT en general por el soporte de esta investigación.

\section{Referencias}

1. Sgardoni V., Nix Andrew R.: Raptor Code-Aware Link Adaptationfor Spectrally Efficient Unicast VideoStreaming over Mobile Broadband Networks, IEEE Transactions on Mobile Computing, Vol. 14, No. 2, pp. 401-415 (2015)

2. Li Bo, Wang Peng, Zhang Yongfei: Classification-based Multi-client Video. Journal of Multimedia, Vol. 8, No. 4, pp. 315-323.

3. TRICALCAR. www.wilac.net/tricalcar (2015)

4. Yuan-Hui, $\mathrm{Yu}$, Chin-Chen Chang, Yu-Chen Hu: Hiding secret data in images via predictive coding. Pattern Recognition, 38, pp. 691-705 (2005)

5. Carvajal-Gámez, B.E., Gallegos-Funes, F.J., López-Bonilla, J.L.: Método simple de ocultamiento de datos para imágenes RGB basado en Estimación de la Varianza. In: COMIA (2012)

6. Carvajal-Gamez, B.E., Gallegos-Funes, F.J., Rosales-Silva, A.J.: Color local complexity estimation based steganographic (CLCES) method. Expert Systems with Applications, 40, pp. 1132-1142 (2013)

7. Yesilyurt, M., Yalman, Y., Turan Ozcerit, A.: A Robust Watermarking Method for Mpeg4 Based on Kurtosis. The Computer Journal, doi: 10.1093/comjnl/bxu112 (2014)

8. MoccagattaIole, T. R.: MPEG-4 video verification model: Status and directions. International Journal of Imaging Systems and Technology, Vol. 8, No. 5, pp. 468-479 (1997)

9. Daubechies, I.: Orthornormal bases of compactly supported wavelets. Communications on Pure Applied Mathematics, 41, pp. 909-996 (1988) 
10. Debnath, L.: Wavelets and Signal Processing. Birkhauser, Berlin, 106 p. (2002)

11. Ginesu, G, Massidda, F., Giusto, D.D.: A multi-factors approach for image quality assessment based on a human visual system model. Signal Processing: Image communication, Vol. 21, No. 4, pp. 316-333 (2006)

12. Huang, K.Q., Wu, Z.Y., Fung, G.S.K., Chan, H.Y.: Color image denoising with wavelet thresholding based on human visual system model. Signal Processing: Image communication, Vol. 20, No. 2, pp. 115-127 (2005)

13. Carvajal-Gámez, B. E., Gallegos-Funes, F. J., Rosales-Silva, A. J., López-Bonilla J. L.: Adjust of energy with compactly supported orthogonal wavelet for steganographic algorithms using the scaling function $1 / \sqrt{2^{j}}$. International Journal of Physical Sciences, Vol. 8, No. 4, pp. 157-166, doi: 10.5897/IJPS12.516 (2013)

14. Chan, C. K., Cheng, L. M.: Hiding data in images by simple LSB substitution. Pattern Recognition, vol. 37, pp. 469-474 (2004)

15. Yu, Y. H., Chang, C. C., Lin, I. C.: A new steganographic method for color and grayscale image hiding. Computer Vision and Image Understanding, Vol. 107, No. 3, pp. 183-194 (2007)

16. Cortés Hernández, G. A.: Cómo calcular el ancho de banda de un enlace para video. Primera parte, ww.rnd.com.ar (2015) 